\title{
Association between Leukocyte Mitochondrial DNA Copy Number and Regular Exercise in Postmenopausal Women
}

\author{
Yu Kyung Chang, Da Eun Kim, Soo Hyun Cho, Jung-Ha Kim* \\ Department of Family Medicine, Chung-Ang University Hospital, Chung-Ang University College of Medicine, Seoul, Korea
}

\begin{abstract}
Background: Previous studies suggest that habitual exercise can improve skeletal mitochondrial function; however, to date, the association between exercise and mitochondrial function in peripheral leukocytes has not been reported. The aim of this study was to evaluate the relationship between regular exercise and mitochondrial function by measuring leukocyte mitochondrial DNA (mtDNA) copy number in postmenopausal women.

Methods: This cross-sectional study included 144 relatively healthy, non-diabetic, non-smoking, postmenopausal women. Clinical parameters, including anthropometric measurements and cardio-metabolic parameters, were assessed. Regular exercise was defined as at least 150 minutes per week of moderate-intensity activity, or an equivalent combination of moderate and vigorous-intensity activity, over a duration of at least 6 months. Leukocyte mtDNA copy numbers were measured using real-time polymerase chain reaction assays, and these were normalized to the $\beta$-globin copy number to give the relative mtDNA copy number.

Results: The mtDNA copy number of peripheral leukocytes was significantly greater in the exercise group $(1.33 \pm 0.02)$ than in the no exercise group $(1.05 \pm 0.02, \mathrm{P}<0.01)$. Stepwise multiple regression analysis showed that regular exercise was independently associated with mtDNA copy number $(\beta=0.25, \mathrm{P}<0.01)$ after adjusting for the variables age, body mass index, waist-to-hip ratio, systolic and diastolic blood pressure, homeostasis model assessment of insulin resistance value, and levels of high-density lipoprotein cholesterol, triglycerides, and homocysteine. Conclusion: Regular exercise is associated with greater leukocyte mtDNA copy number in postmenopausal women.
\end{abstract}

Keywords: Leukocytes; Mitochondria; DNA Copy Number; Exercise; Postmenopausal 


\section{INTRODUCTION}

Mitochondria provide energy for cellular processes by generating adenosine 5-triphosphate (ATP) via the electron transport chain. ${ }^{1)}$ Additionally, they are important in the regulation of immune responses to both pathogenic infections and oxidative stress, ${ }^{2)}$ and they are essential for the maintenance of homeostatic mechanisms such as cell survival, redox signaling, and death. ${ }^{3)}$ Mitochondrial DNA (mtDNA) copy number, which is proportional to the volume of mtDNA in a cell, is frequently used as a measure of mitochondrial function. ${ }^{4,5)}$

Characteristics such as muscle mass and strength, cardiovascular fitness, and respiratory function are all enhanced by regular exercise, ${ }^{6,7)}$ which can also improve mitochondrial function in skeletal muscle., Additionally, regular exercise is known to inhibit both the ageing process and the progression of several age-related diseases. While the underlying mechanisms are not fully elucidated, anti-oxidative and antiinflammatory effects are known to play a role. ${ }^{3,8)}$ In leukocytes, important immune system components that are essential for inflammatory responses, the mitochondrial transmembrane potential, a functional marker of energy status and viability, is thought to be affected by regular exercise. ${ }^{9,10)}$ It is possible that regular activity enhances the immune system and attenuates inflammatory responses by enhancing leukocyte mitochondrial functions, ${ }^{11)}$ but, to date, no study of this relationship in peripheral lymphocytes has been reported.

The aim of this study was to evaluate the relationship between regular exercise and mitochondrial function by measuring the mtDNA copy number in leukocytes from postmenopausal women. The selection of these particular study participants minimized the effects of estrogen on the biogenesis of mitochondria and, subsequently, their effects on anti-oxidative and anti-inflammatory pathways. ${ }^{12)}$

\section{METHODS}

\section{Study Design and Participants}

This cross-sectional study included 144 relatively healthy, non-diabetic, non-smoking, postmenopausal women who visited the primary healthcare clinic of the Chung-Ang University Hospital between October 2009 and May 2010. None of the participants had menstruated for over a year, and none were being treated with any hormonal therapy. Medical records were used to collect data regarding previous and current medical conditions and medications taken. Individuals with a history of cardiovascular disease, including myocardial infarction, transient ischemic attack, or cerebral infarction, were excluded from the study.

Regular exercise was defined as at least 150 minutes per week of moderate-intensity activity, or an equivalent combination of moderate and vigorous-intensity activity, ${ }^{13)}$ over a duration of at least 6 months. The no exercise group comprised the study participants who did not meet these criteria. Alcohol consumption was defined as the consumption of at least $72 \mathrm{~g}$ of alcohol per week. ${ }^{14)}$ This study was approved by the institutional review board of the Chung-Ang University
Hospital (IRB number C2009051 [238]). All participants provided written informed consent.

\section{Anthropometric Evaluation}

Anthropometric factors, including body weight and height, were obtained by a single, well-trained examiner and were measured to the nearest tenth of the appropriate unit. Weight $(\mathrm{kg})$ and height $(\mathrm{cm})$ were measured using DS 101 (Dongsahn Jenix, Seoul, Korea), and subjects were dressed in light clothing without shoes. Body mass index (BMI) was calculated as weight divided by the square of the height $\left(\mathrm{kg} / \mathrm{m}^{2}\right)$. Blood pressure was measured after resting for 10 minutes in a seated position. Waist circumference $(\mathrm{cm})$ was measured at the midpoint between the superior margin of the iliac crest and the inferior margin of the last rib in a horizontal plane. Waist-to-hip ratio (WHR) was estimated by the InBody 720 body composition analyzer (Biospace, Seoul, Korea).

\section{Cardio-Metabolic Parameter Assay}

All blood samples were obtained after 12 hours of overnight fasting. Serum levels of uric acid, glucose, total cholesterol, high-density lipoprotein cholesterol (HDL), low-density lipoprotein cholesterol (LDL), triglycerides, and high-sensitivity C-reactive protein (hs-CRP) were measured using the ADVIA 1650 chemistry analyzer (Siemens, Munich, Germany). Levels of fasting insulin were measured using an electrochemiluminescence immunoassay system according to the supplier's protocol (Roche, Indianapolis, IN, USA). Insulin resistance was estimated using the homeostasis model assessment of insulin resistance (HOMA-IR) index, defined as follows:

HOMA-IR=(insulin $(\mu \mathrm{IU} / \mathrm{mL}) \times$ fasting blood glucose $(\mathrm{mg} / \mathrm{dL}) / 18) / 22.5$

Plasma concentrations of adiponectin and homocysteine were measured using either AdipoGen (Seoul, Korea) or Immulite 2000 (Siemens) immunoassay kits, respectively.

\section{Measurement of Peripheral Blood Leukocyte Mitochondrial DNA Copy Number}

mtDNA was extracted from the peripheral leukocytes in $1 \mathrm{~mL}$ of whole blood using a QIAamp DNA kit (Qiagen, Valencia, CA, USA). The mtDNA copy number was determined by real-time polymerase chain reaction, using the LightCycler FastStart DNA Master SYBR Green I kit according to the manufacturer's instructions (Roche, Pleasanton, CA, USA). The quantity of mtDNA was normalized to the expression of nuclear $\beta$-globin. Forward and reverse primers for the $\beta$-globin gene were 5'-GAAGAGCCAAGGACAGGTAC-3' and 5'-CAACTTCATCCACGTTCACC-3, respectively, and forward and reverse primers for the mitochondrial ND1 gene were 5'-AACATACCCATGGCCAACCT-3' and 5'-AGCGAAGGGTTGTAGTAGCCC-3', respectively. After denaturation at $95^{\circ} \mathrm{C}$ for 300 seconds, samples were subjected to 40 cycles of incubation at $95^{\circ} \mathrm{C}$ for 0.1 seconds, $58^{\circ} \mathrm{C}$ for 6 seconds, and $72^{\circ} \mathrm{C}$ for 18 seconds. The threshold cycle number $(\mathrm{Ct})$ was defined as the number 
of polymerase chain reaction cycles needed to produce $20 \mathrm{ng}$ of DNA product. The mtDNA copy number was calculated using the following equation: relative copy number $=2^{\Delta \mathrm{Ct}}\left(\Delta \mathrm{Ct}=\mathrm{Ct}_{\beta \text {-globin }}-\mathrm{Ct}_{\mathrm{ND1}}\right)$.

\section{Statistical Analysis}

Data are presented as mean \pm standard deviation or number (n, \%). The significance of any differences in baseline characteristics between the two groups were assessed using either a t-test or a chi-square or Fisher's exact test, for continuous variables or categorical variables, respectively. The leukocyte mtDNA copy numbers, the concentrations of adiponectin, hs-CRP, insulin, and homocysteine, and the HOMA-IR values were logarithmically transformed to approximate a normal distribution before statistical analyses were performed. An independent association between leukocyte mtDNA copy number and exercise was confirmed using a stepwise multiple linear regression model with a significance threshold of 0.15 . Potential confounding variables were defined as those that were significantly associated $(\mathrm{P}<0.1)$ with leukocyte mtDNA copy number using Pearson's correlation analysis, either in this study (triglyceride, WHR, waist circumference, and homocysteine level), or in previously reported studies (age, ${ }^{15)} \mathrm{BMI},{ }^{16)}$ systolic and diastolic blood pressure, HOMA-IR, and levels of triglyceride and HDL cholesterol $^{17,18)}$ ). Multicollinearity between independent variables was evaluated using Pearson's correlation coefficients. If baseline variables were correlated ( $r>0.6$ and $\mathrm{P}<0.05$ ), only one variable was included in the analysis; for instance, as WHR and waist circumference showed multicollinearity, waist circumference was excluded from the analysis. The leukocyte mtDNA copy numbers of the exercise and no exercise groups, presented as the least squares mean \pm standard error, were compared using the analysis of covariance model while adjusting for age and WHR. All statistical analyses were performed using the SAS ver. 9.1 statistics package (SAS Institute Inc., Cary, NC, USA).

\section{RESULTS}

\section{Clinical Characteristics of the Study Subjects}

The mean age of the participants was $57.4 \pm 6.1$ years, and ranged from 50 to 72 years. The clinical characteristics of the study participants in the no exercise and regular exercise groups are shown in Table 1. Waist circumference, WHR, and triglyceride level were all significantly higher in the no exercise group than in the regular exercise group $(\mathrm{P}<0.01)$. Conversely, no significant differences in age, BMI, blood pressure, alcohol consumption, antihypertensive medication use, or concentrations of fasting glucose, total cholesterol, HDL cholesterol, LDL cholesterol, uric acid, log-transformed hs-CRP, homocysteine, adiponectin, or fasting insulin, HOMA-IR were observed between the groups.

\section{Relationship between Regular Exercise and Mitochondrial DNA Copy Number}

Peripheral leukocyte mtDNA copy number was significantly greater in the regular exercise group $(1.33 \pm 0.02)$ than in the no exercise group $(1.05 \pm 0.02, \mathrm{P}<0.01)$ (Figure 1).

\section{Multiple Linear Regression Analysis of the Independent Relationship between Peripheral Leukocyte Mitochondrial DNA Copy Number and Regular Exercise}

Table 2 shows the independent association between exercise and mtDNA copy number in peripheral leukocytes. Stepwise multiple regression analysis showed that regular exercise was independently as-

Table 1. Clinical characteristics of the participants

\begin{tabular}{|c|c|c|c|c|}
\hline Characteristic & Total $(n=144)$ & No exercise $(n=94)$ & Regular exercise $(n=50)$ & P-value \\
\hline Age (y) & $57.4 \pm 6.1$ & $57.7 \pm 6.1$ & $56.7 \pm 6.1$ & 0.35 \\
\hline Body mass index $\left(\mathrm{kg} / \mathrm{m}^{2}\right)$ & $24.5 \pm 2.6$ & $24.6 \pm 2.7$ & $24.3 \pm 2.5$ & 0.45 \\
\hline Waist circumference $(\mathrm{cm})$ & $82.3 \pm 6.9$ & $83.8 \pm 7.0$ & $79.6 \pm 5.9$ & $<0.01$ \\
\hline Waist-to-hip ratio & $0.9 \pm 0.1$ & $0.9 \pm 0.1$ & $0.8 \pm 0.0$ & $<0.01$ \\
\hline Systolic blood pressure (mm Hg) & $123.2 \pm 14.0$ & $123.8 \pm 14.8$ & $122.8 \pm 13.7$ & 0.67 \\
\hline Diastolic blood pressure $(\mathrm{mm} \mathrm{Hg})$ & $72.4 \pm 9.0$ & $72.7 \pm 9.8$ & $72.2 \pm 8.7$ & 0.73 \\
\hline Fasting glucose (mg/dL) & $88.7 \pm 18.9$ & $90.0 \pm 21.0$ & $86.4 \pm 13.9$ & 0.23 \\
\hline Fasting insulin $(\mu \mathrm{IU} / \mathrm{mL})^{*}$ & $6.0 \pm 4.8$ & $6.5 \pm 5.5$ & $5.1 \pm 2.7$ & 0.09 \\
\hline Homeostasis model assessment of insulin resistance* & $1.4 \pm 1.6$ & $1.6 \pm 1.9$ & $1.1 \pm 0.7$ & 0.14 \\
\hline Total cholesterol (mg/dL) & $208.0 \pm 36.4$ & $209.9 \pm 35.8$ & $204.5 \pm 37.5$ & 0.40 \\
\hline Triglyceride (mg/dL) & $130.7 \pm 102.6$ & $148.1 \pm 116.7$ & $97.9 \pm 56.7$ & $<0.01$ \\
\hline High-density lipoprotein cholesterol (mg/dL) & $56.0 \pm 12.0$ & $55.4 \pm 12.3$ & $57.0 \pm 11.4$ & 0.46 \\
\hline Low-density lipoprotein cholesterol (mg/dL) & $125.9 \pm 33.4$ & $124.8 \pm 33.4$ & $127.9 \pm 33.7$ & 0.60 \\
\hline Uric acid (mg/dL) & $3.9 \pm 0.9$ & $3.9 \pm 0.9$ & $4.0 \pm 0.9$ & 0.49 \\
\hline High-sensitivity C-reactive protein $(\mathrm{mg} / \mathrm{mL})^{*}$ & $0.2 \pm 0.4$ & $0.2 \pm 0.6$ & $0.1 \pm 0.2$ & 0.95 \\
\hline Homocysteine $(\mu \mathrm{mol} / \mathrm{L})^{*}$ & $12.0 \pm 9.1$ & $12.6 \pm 11.2$ & $10.8 \pm 1.7$ & 0.07 \\
\hline Adiponectin* & $6.4 \pm 4.3$ & $6.4 \pm 4.4$ & $6.5 \pm 4.0$ & 0.77 \\
\hline Alcohol consumption, $n(\%)^{\dagger}$ & $8(5.6)$ & $4(4.3)$ & $4(8.0)$ & 0.45 \\
\hline Antihypertensive medication, $\mathrm{n}(\%)^{\dagger}$ & $11(7.6)$ & $6(6.4)$ & $5(10.0)$ & 0.51 \\
\hline
\end{tabular}

Values are presented as mean \pm standard deviation or number (\%). P-values were calculated using a t-test, chi-square test, or Fisher's exact test to compare the no exercise and regular exercise groups.

*Values were analyzed after log transformation. ' $\mathrm{N}$ meant the 'number of participants' and the value in parentheses is the percentage among the total participants. 


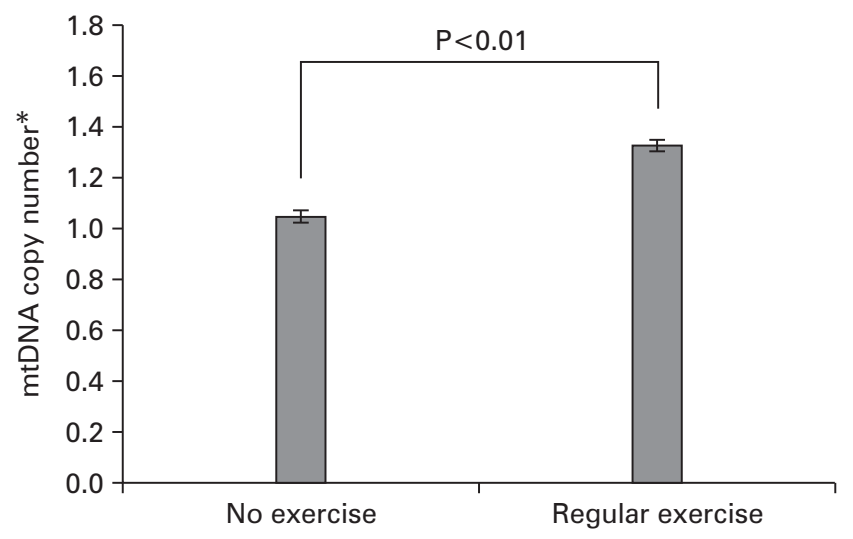

Figure 1. mtDNA copy numbers in the peripheral blood leukocytes of postmenopausal women who either exercised regularly or had a sedentary lifestyle. The mtDNA copy number was significantly higher in the regular exercise group than in the no exercise group. P-values were calculated by analysis of covariance (adjusted for age and waist-to-hip ratio). mtDNA, mitochondrial DNA. *Logtransformed, mtDNA copy number normalized to $\beta$-globin gene copy number.

sociated with $m$ tDNA copy number $(\beta=0.25, \mathrm{P}<0.01)$ after adjusting for age, BMI, WHR, systolic and diastolic blood pressure, HDL cholesterol level, triglyceride level, HOMA-IR, and homocysteine level.

\section{DISCUSSION}

In this cross-sectional study, we have used a stepwise multiple regression analysis, adjusting for potential confounders such as age, ${ }^{15)} \mathrm{BMI}{ }^{16)}$ and various cardio-metabolic parameters, ${ }^{17,18)}$ to show that postmenopausal women who partake in regular exercise have a significantly higher leukocyte mtDNA copy number than postmenopausal women who do not exercise.

Nonetheless, a negative relationship between peripheral leukocyte mtDNA copy number and age was observed, which is consistent with the previously reported age-associated decline in mitochondrial function in various cell types, including skeletal muscle ${ }^{4,7,19)}$ and leukocytes. $^{15,20)}$

While the cross-sectional design of the study means that neither a causal relationship between regular exercise and mtDNA copy number nor any specific mechanisms underlying the association can be determined, potential mechanisms may still be suggested.

Firstly, the anti-oxidative and anti-inflammatory effects of regular exercise may affect leukocyte mtDNA copy number. Mitochondria are the primary generators of reactive oxygen species, ${ }^{1)}$ and so are themselves at greater risk of oxidative injury, resulting in a reduction in cellular mitochondrial content. ${ }^{21)}$ Alterations in the expression of pro-inflammatory and oxidant genes and transcription factors during habitual exercise have been reported in mononuclear cells. ${ }^{22)}$ Pro-inflammatory cytokines such as tumor necrosis factor- $\alpha$ (TNF- $\alpha$ ), nuclear factor- ${ }_{\kappa} \mathrm{B}\left(\mathrm{NF}-{ }_{\mathrm{K}} \mathrm{B}\right)$, and monocyte chemoattractant protein-1 were shown to be upregulated during the acute phase of exercise, with the effect being largely ameliorated by habitual exercise. ${ }^{22)}$ Moreover, a
Table 2. Stepwise multiple linear regression analysis to identify clinical variables associated with mitochondrial DNA copy number

\begin{tabular}{lcccc}
\hline \multicolumn{1}{c}{ Variable } & \multicolumn{1}{c}{$\boldsymbol{\beta}$} & Standard error & F-value & P-value \\
\hline Exercise & 0.25 & 0.03 & 97.15 & $<0.01$ \\
Triglyceride & $-<0.01$ & $<0.01$ & 13.56 & $<0.01$ \\
Age & -0.01 & $<0.01$ & 6.83 & $<0.01$ \\
Diastolic blood pressure & $-<0.01$ & $<0.01$ & 3.01 & 0.08 \\
Waist-to-hip ratio & -0.41 & 0.25 & 2.62 & 0.11 \\
\hline
\end{tabular}

$R^{2}=52.4$. The variables considered initially were age, body mass index, waist-to-hip ratio, systolic and diastolic blood pressure, HOMA-IR, levels of high-density lipoprotein cholesterol, triglycerides, and homocysteine. The variables selected for entry into the model, shown above, were those that reached the 0.15 significance threshold. HOMA-IR and homocysteine were analyzed after log transformation. HOMA-IR, homeostasis model assessment of insulin resistance.

randomized controlled study showed that indicators of systemic inflammation, such as the concentration of hs-CRP, TNF- $\alpha$, white blood cells, and plasma F2-isoprostane, were significantly lower in the frequent exercise group than in the control group. ${ }^{23)}$ Such anti-oxidative and anti-inflammatory effects of regular exercise were seen consistently in skeletal muscle, ${ }^{8)}$ and mild to moderate exercise was found to generate reactive oxygen species, which then activated mitochondrial responses and improved mitochondrial quality in skeletal muscle. Components of biosynthetic pathways in mitochondria are constantly exchanged during biogenesis, fusion/fission events, and quality proofreading. ${ }^{7)}$

Secondly, the association between regular exercise and leukocyte mtDNA copy number may be related to lipolysis, which is known to increase with regular exercise. ${ }^{24)}$ Lipolysis is the generation of free fatty acids (FFAs), a metabolic substrate, from the catabolism of triacylglycerol. FFAs are not only an essential substrate for energy production, but are also important ligands for cell signaling via the peroxisome proliferator-activated receptor (PPAR)/peroxisome proliferator-activated receptor gamma coactivator-1 alpha (PGC-1 $\alpha$ ) complex. ${ }^{7,25)}$ PGC-1 $\alpha$ is important for mitochondrial biogenesis and the destruction of defective mitochondria, ${ }^{7)}$ and is known to be more active under chronic exercise conditions. This higher activity results in the upregulation of genes such as mitochondrial transcription factor A and protein import machinery components, giving higher mtDNA gene expression, greater oxygen capacity, and increased ATP synthesis in skeletal muscle. ${ }^{3)}$ PGC-1 $\alpha$ is also found in peripheral leukocytes, wherein its expression was shown to be elevated after exercise. ${ }^{26)}$

Finally, regular exercise may impair the attrition of telomeres, the nucleoprotein complexes found at the ends of chromosomes that function to maintain chromosomal stability, and was shown to be beneficial for leukocyte telomere length. ${ }^{14)}$ Telomeres are known to influence mitochondrial functions, including biogenesis and metabolism, in various cells and organs, ${ }^{27)}$ and telomere length has been positively associated with mtDNA copy number in peripheral leukocytes. ${ }^{14)}$ It is thus possible that regular exercise increases mtDNA copy number by maintaining telomere length.

To the best of our knowledge, this is the first report of the relation- 
ship between regular exercise and leukocyte mtDNA copy number, a proxy for mitochondrial function, in postmenopausal women.

However, this study does have some limitations. Firstly, the crosssectional study design meant that causal relationships between $\mathrm{mtD}$ NA copy number and physical exercise could not be determined. Secondly, there may be some selection bias in choosing the study participants, as all were patients visiting an outpatient clinic of a hospital. Thirdly, the level of circulating estradiol in the participants was not measured; however, because the women participating in the study had been postmenopausal for at least 1 year, it is reasonable to assume that the effect of serum estradiol was negligible.

In conclusion, leukocyte mtDNA copy number may be associated with regular exercise in postmenopausal women. Our study suggests that regular exercise may improve leukocyte mitochondria function, and thus be of benefit in the ageing process as well as in several inflammatory conditions.

\section{CONFLICT OF INTEREST}

No potential conflict of interest relevant to this article was reported.

\section{CONTRIBUTORS AND ROLES}

Study concept (hypothesis) and design: Yu Kyung Chang, Da Eun Kim, Jung-Ha Kim

Analysis and interpretation of data: Yu Kyung Chang, Jung-Ha Kim

Preparation of the manuscript: Yu Kyung Chang, Soo Hyun Cho, JungHa Kim

Review of the manuscript: Yu Kyung Chang, Da Eun Kim, Soo Hyun Cho, Jung-Ha Kim

\section{REFERENCES}

1. McBride HM, Neuspiel M, Wasiak S. Mitochondria: more than just a powerhouse. Curr Biol 2006;16:R551-60.

2. Weinberg SE, Sena LA, Chandel NS. Mitochondria in the regulation of innate and adaptive immunity. Immunity 2015;42:406-17.

3. Carter HN, Chen CC, Hood DA. Mitochondria, muscle health, and exercise with advancing age. Physiology (Bethesda) 2015;30:208-23.

4. Short KR, Bigelow ML, Kahl J, Singh R, Coenen-Schimke J, Raghavakaimal S, et al. Decline in skeletal muscle mitochondrial function with aging in humans. Proc Natl Acad Sci U S A 2005;102:5618-23.

5. Clay Montier LL, Deng JJ, Bai Y. Number matters: control of mammalian mitochondrial DNA copy number. J Genet Genomics 2009;36:12531.

6. Cobley JN, Moult PR, Burniston JG, Morton JP, Close GL. Exercise improves mitochondrial and redox-regulated stress responses in the elderly: better late than never! Biogerontology 2015;16:249-64.

7. Barbieri E, Agostini D, Polidori E, Potenza L, Guescini M, Lucertini F, et al. The pleiotropic effect of physical exercise on mitochondrial dynamics in aging skeletal muscle. Oxid Med Cell Longev 2015;2015:917085.

8. Sallam N, Laher I. Exercise modulates oxidative stress and inflammation in aging and cardiovascular diseases. Oxid Med Cell Longev
2016;2016:7239639.

9. Hsu TG, Hsu KM, Kong CW, Lu FJ, Cheng H, Tsai K. Leukocyte mitochondria alterations after aerobic exercise in trained human subjects. Med Sci Sports Exerc 2002;34:438-42.

10. Kulkarni OP, Lichtnekert J, Anders HJ, Mulay SR. The immune system in tissue environments regaining homeostasis after injury: is "inflammation” always inflammation? Mediators Inflamm 2016;2016:2856213.

11. Kruger K, Mooren FC. Exercise-induced leukocyte apoptosis. Exerc Immunol Rev 2014;20:117-34.

12. Gupte AA, Pownall HJ, Hamilton DJ. Estrogen: an emerging regulator of insulin action and mitochondrial function. J Diabetes Res 2015;2015:916585.

13. Centers for Disease Control and Prevention. Physical activity guidelines for Americans fact sheet for health professionals [Internet]. Atlanta (GA): Centers for Disease Control and Prevention; 2008 [cited 2016 Nov 8]. Available from: http://www.cdc.gov/physicalactivity/ downloads/pa_fact_sheet_adults.pdf.

14. Kim JH, Ko JH, Lee DC, Lim I, Bang H. Habitual physical exercise has beneficial effects on telomere length in postmenopausal women. Menopause 2012;19:1109-15.

15. Kim JH, Kim HK, Ko JH, Bang H, Lee DC. The relationship between leukocyte mitochondrial DNA copy number and telomere length in community-dwelling elderly women. PLoS One 2013;8:e67227.

16. Meng S, Wu S, Liang L, Liang G, Giovannucci E, Vivo I, et al. Leukocyte mitochondrial DNA copy number, anthropometric indices, and weight change in US women. Oncotarget 2016 Jun 29 [Epub]. https:// doi.org/10.18632/oncotarget.10325.

17. Huang CH, Su SL, Hsieh MC, Cheng WL, Chang CC, Wu HL, et al. Depleted leukocyte mitochondrial DNA copy number in metabolic syndrome. J Atheroscler Thromb 2011;18:867-73.

18. Xu FX, Zhou X, Shen F, Pang R, Liu SM. Decreased peripheral blood mitochondrial DNA content is related to HbAlc, fasting plasma glucose level and age of onset in type 2 diabetes mellitus. Diabet Med 2012;29:e47-54.

19. Tonkonogi M, Sahlin K. Physical exercise and mitochondrial function in human skeletal muscle. Exerc Sport Sci Rev 2002;30:129-37.

20. Mengel-From J, Thinggaard M, Dalgard C, Kyvik KO, Christensen K, Christiansen L. Mitochondrial DNA copy number in peripheral blood cells declines with age and is associated with general health among elderly. Hum Genet 2014;133:1149-59.

21. Liu CS, Tsai CS, Kuo CL, Chen HW, Lii CK, Ma YS, et al. Oxidative stress-related alteration of the copy number of mitochondrial DNA in human leukocytes. Free Radic Res 2003;37:1307-17.

22. Gano LB, Donato AJ, Pierce GL, Pasha HM, Magerko KA, Roeca C, et al. Increased proinflammatory and oxidant gene expression in circulating mononuclear cells in older adults: amelioration by habitual exercise. Physiol Genomics 2011;43:895-902.

23. Campbell PT, Gross MD, Potter JD, Schmitz KH, Duggan C, McTiernan A, Ulrich CM. Effect of exercise on oxidative stress: a 12-month randomized, controlled trial. Med Sci Sports Exerc 2010;42:1448-53.

24. Huang CJ, Kwok CF, Chou CH, Chou YC, Ho LT, Shih KC. The effect of exercise on lipid profiles and inflammatory markers in lean male adolescents: a prospective interventional study. J Investig Med 2015;63:2934.

25. Kratky D, Obrowsky S, Kolb D, Radovic B. Pleiotropic regulation of mi- 
tochondrial function by adipose triglyceride lipase-mediated lipolysis. Biochimie 2014;96:106-12.

26. Ferrer MD, Tauler P, Sureda A, Tur JA, Pons A. Antioxidant regulatory mechanisms in neutrophils and lymphocytes after intense exercise. J
Sports Sci 2009;27:49-58.

27. Sahin E, Colla S, Liesa M, Moslehi J, Muller FL, Guo M, et al. Telomere dysfunction induces metabolic and mitochondrial compromise. Nature 2011;470:359-65. 